\title{
Extending the Design Space of Tangible Objects via Low-Resolution Edge Displays
}

\author{
Ahmed Sabbir Arif ${ }^{1}$, Brien East ${ }^{1}$, Sean DeLong ${ }^{1}$ \\ Roozbeh Manshaei ${ }^{1}$, Apurva Gupta ${ }^{2}$, \\ Manasvi Lalwani ${ }^{2}$, Ali Mazalek ${ }^{1,2}$ \\ Synaesthetic Media Laboratory \\ ${ }^{1}$ Ryerson University \\ Toronto, Ontario, Canada \\ a.s.arif@gmail.com, \{beast, sean.delong, \\ roozbeh.manshaei\}@ryerson.ca \\ ${ }^{2}$ Georgia Institute of Technology \\ Atlanta, Georgia, USA \\ \{apurva.gupta, manasvi.lalwani, mazalek\}@gatech.edu
}

Permission to make digital or hard copies of part or all of this work for personal or classroom use is granted without fee provided that copies are not made or distributed for profit or commercial advantage and that copies bear this notice and the full citation on the first page. Copyrights for third-party components of this work must be honored. For all other uses, contact the Owner/Author.

uses, contact the Owner/Author.
Copyright is held by the owner/author(s).

TEI '17, March 20-23, 2017, Yokohama, Japan

ACM $978-1-4503-4676-4 / 17 / 03$.

http://dx.doi.org/10.1145/3024969.3025078

\begin{abstract}
We developed a custom tangible that uses LED arrays around the edges as a low-resolution display to provide real-time visual feedback on the current state of the system. We developed a guideline for mapping different types of edge feedback to different tangible interactions. We evaluated its effectiveness in an informal user study where users interacted with a tabletop and tangible system with the edge feedback enabled. Results suggest that edge feedback provides a better understanding of the system.
\end{abstract}

\section{Author Keywords}

LEDs; point lights; active/passive tangibles; feedback.

\section{ACM Classification Keywords}

H.5.m. Information interfaces and presentation (e.g., $\mathrm{HCI}$ ): Miscellaneous.

\section{Introduction}

Brygg et al. [26] defined tangibles, also known as tokens, as "discrete, spatially reconfigurable physical objects that typically represent digital information". A tangible could be either passive or active. Passive tangibles employ a one-way communication model, typically from the tangibles to other interactive devices, hence cannot reflect changes in the digital model. Active tangibles, on the other hand, maintain a two- 


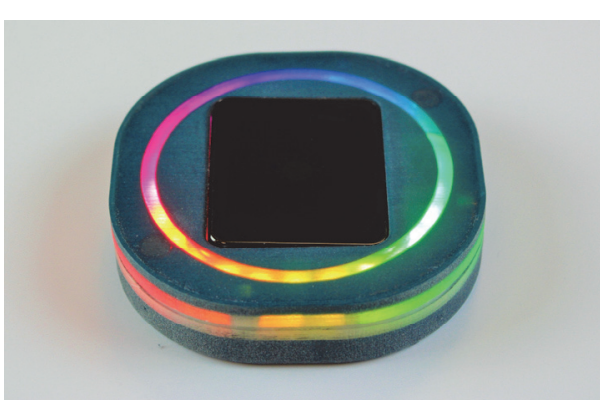

Figure 1. An active tangible augmented with low-resolution edge display (i.e., LED array). way communication between the tangibles and other devices, therefore can reflect changes in the digital model. Passive or active tangibles, together with other interactive devices, form a tangible user interface [26].

Feedback is an important part of interactive systems. It communicates the results of an interaction, making it visible and understandable. Feedback answers questions across four categories, specifically the user's location on a chain of tasks, current state of the system, future state of the system, and the outcome of an action [19]. Feedback not only informs users of whether they are moving closer to accomplishing a task or not but also when errors occur and how to address them.

Prior studies showed that feedback can provide users with a better understanding of the system, facilitating a swift transition from novice to expert [14]. Yet most current tangibles are passive and do not provide any feedback on tangible interactions [22]. They usually rely on an additional device to provide feedback (i.e., an interactive tabletop), confining the interaction space within the proximity of that device. Some tangibles provide visual feedback on the display, when available, i.e., notification window, but this feedback often occludes the information on the screen. It also typically requires a user action upon receipt (i.e., a tap to discard a notification window) affecting the natural flow of the task at hand [2]. In this paper, we propose augmenting tangibles with a low-resolution edge display (Figure 1) to provide real-time visual feedback on user interactions and the current state of the system.

The remainder of the paper is organized as follows. We start with a literature review of existing work exploring non-graphical displays for visual feedback. We then discuss the motivation of the work, and propose a guideline for mapping different types of edge feedback to different tangible interactions. We then apply it to an existing tabletop and tangible system to demonstrate its effectiveness. We evaluate the system in an informal investigation. Finally, we conclude with speculation on future extensions to the work.

\section{Related Work}

Harrison et al. [10] explored the types of information conveyed by a single-color point light in current devices, and investigated whether their design space could be enriched by using varying light intensity over time. They identified twenty-four different light behaviors, and based on an evaluation, recommended eight of them to use in a mobile device domain (e.g., different beacon, flash, brightness, pulse, and blinking behaviors). $\mathrm{Xu}$ and Lyons [30] developed two smartwatch prototypes to demonstrate that low-resolution edge feedback could offer smart capabilities. The first prototype was augmented with four LEDs in the four directions and the second was augmented with twelve LEDs arranged corresponding to the hours on a watch face. Both used different colors, brightness, and blinking patterns to provide users with feedback on different smartwatch apps and their respective parameters.

Kanis et al. [12] augmented a bracelet with a $5 \times 5$ LED array that automatically exchanged information between users when they shook hands. It created a cycling animation on each successful data transfer episode. Similarly, Williams et al. [28] designed a digital bracelet for remote text messaging. It included six studs in a row, each containing four LEDs in red, blue, green, and white. Five of the studs represented individuals and one represented whole-group activity. It used a pulsing 
white LED to indicate that a message is waiting from the corresponding individual. The colored LEDs glowed to convey a specific type of message (i.e., availability of a user), preset by the members of the group.

In a different study, Tarasewich et al. [25] investigated how much information could be encoded and understood by users in visual displays ranging in size from two to nine LEDs and using different display characteristics, such as colors and blinking patterns. Similarly, Campbell and Tarasewich [5] studied how much information could be realistically presented on a pixel-based microsized display. Both investigations revealed that users can quickly learn a relatively large number of feedback with a few LEDs and their performance and preference increase with multiple LEDs and effective display designs.

Visual feedback on tangible objects is not as well explored. Almost all current tangibles provide visual feedback on an integrated display screen when available $[3,13,15,16]$ or on an external display, such as an interactive wall $[8,24]$ and interactive tabletops $[23,29]$. Some have also explored lights [11] and other forms of feedback, such as haptic [20-22] and auditory [4]. Several tangibles provide visual feedback through a small number of LEDs $[6,17,27]$. However, no prior work has explored the full potential of low-resolution edge display in the context of tangibles.

\section{Motivation}

Apart from increasing the user's awareness of the current state of the system, the following potentials of a low-resolution edge display have motivated the work.

Low mental and perceptual demand. In theory, edge feedback should require fewer mental and perceptual activities than other feedback types. Particularly, textual and graphical feedback require users to move their foci from one part of the visual field to another and usually require a user-action upon receipt (i.e., a tap on the screen to remove the notification window). These restrict the possibility of task parallelism and reduce user performance both in terms of speed and accuracy [2]. Auditory feedback, on the other hand, is unreliable in noisy places and relies on an additional sensory organ (i.e., our ears), which increases perceptual demand. Edge feedback is free from most of these limitations.

Swift conversion and transition. Low-resolution edge displays could be added to most active tangibles, allowing designers to incorporate edge feedback in their system. Since the display need not compromise or alter physical design of the tangibles, existing users do not have to learn an entirely new interaction, accommodating a swift transition from old to new tangibles.

Extensibility. Although, we explore edge feedback in the context of active tangibles, low-resolution edge displays could be added to other interactive systems as well, i.e., wearable devices and computer accessories.

Independent interactions. Edge feedback could also extend the support for independent interactions with active tangibles. In a tabletop and tangible system, it could aid users in performing actions off-the-table since they could confirm and verify the actions through the feedback provided on the tangibles, without being reliant on the tabletop display. Some existing tangibles (e.g., Siftables [16]) support independent interactions by providing visual feedback on an integrated display screen. However, this often occludes the information on the screen. Edge feedback is free from this limitation. 


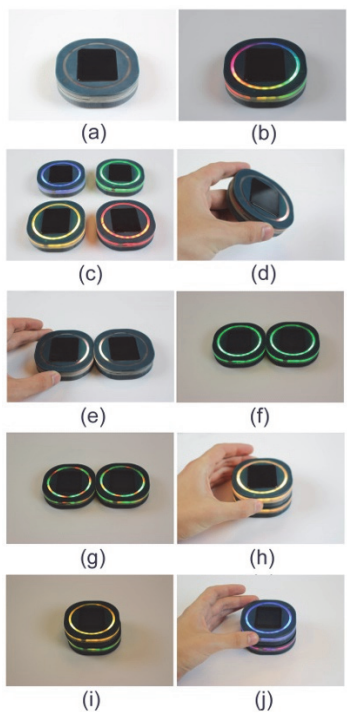

Figure 2. Edge feedback on different tangible interactions: (a) inactive tangible, (b) turned on tangible, (c) tangibles representing different items, (d) tilt notification, (e) neighboring notification, (f) valid neighboring, $(g)$ invalid neighboring, (h) stacking notification, (i) valid stacking, and (j) invalid stacking.
Group interactions. Edge feedback could extend group interactions with tangibles, where users use multiple tangibles to interact with the system. Many systems provide visual feedback on an external display for group interactions (e.g., stacking), which force users to work in a proximity to the display. This also limits independent interactions with the tangibles. Some active tangibles provide visual feedback on integrated display screens. However, when stacked, users could only see the display of the top tangible. Some active tangibles (e.g., Stackables [13]) attach the display on the side to address this, but are not intended for tabletop interaction since the side-screen is difficult

\begin{tabular}{|c|c|c|c|c|}
\hline \# & Manipulation & Interaction & Action & Edge Feedback \\
\hline \multirow[t]{2}{*}{$\begin{array}{l}\text { Single } \\
\text { Tangible }\end{array}$} & $\begin{array}{l}\text { Direct } \\
\text { On/off-the- } \\
\text { table }\end{array}$ & $\begin{array}{l}\text { Changes via tap, } \\
\text { touch, tilt, shake, } \\
\text { flip/rotate, or } \\
\text { tactile buttons }\end{array}$ & $\begin{array}{l}\text { Turn on a tangible } \\
\text { Detection confirmation } \\
\text { Change an item } \\
\text { Change a value } \\
\text { Remove an item } \\
\text { Turn off a tangible }\end{array}$ & $\begin{array}{l}\text { Pulse/blink } k^{1} \text { in multiple colors \& change color to } \text { white }^{3} \\
\text { Blink }{ }^{1} \text { along the edge corresponding to tilt/rotate angle } \\
\text { Change color to represent the new item } \\
\text { Change illumination }{ }^{4} \\
\text { Change color to white } \\
\text { Turn off display }\end{array}$ \\
\hline & Indirect & $\begin{array}{l}\text { Changes via } \\
\text { another device or } \\
\text { system actions }\end{array}$ & $\begin{array}{l}\text { Change an item } \\
\text { Change a value } \\
\text { Remove an item }\end{array}$ & $\begin{array}{l}\text { Change color to represent the new item \& pulse/blink }{ }^{1} \\
\text { Change illumination \& pulse/blink }{ }^{1} \\
\text { Pulse/blink }{ }^{1} \text { \& change color to } \text { white }^{3}\end{array}$ \\
\hline \multirow{2}{*}{$\begin{array}{l}\text { Multiple } \\
\text { Tangibles }\end{array}$} & \multirow{2}{*}{$\begin{array}{l}\text { Direct } \\
\text { On/off-the- } \\
\text { table }\end{array}$} & Neighboring & $\begin{array}{l}\text { Detection confirmation } \\
\text { Valid combination } \\
\text { Invalid combination }\end{array}$ & $\begin{array}{l}\text { Blink }{ }^{1} \text { along the edges that have been neighbored } \\
\text { Change all tangibles to alternating colors } \\
\text { Pulse/blink }{ }^{1} \text { the invalid tangible in color red }{ }^{6}\end{array}$ \\
\hline & & Stacking & $\begin{array}{l}\text { Detection confirmation } \\
\text { Valid combination } \\
\text { Invalid combination }\end{array}$ & $\begin{array}{l}\text { Flash along all edges } \\
\text { Change all tangibles to alternating colors } \\
\text { Pulse/blink }{ }^{1} \text { the invalid tangible in color red }\end{array}$ \\
\hline
\end{tabular}

Pulse or flash rates may differ for different tangibles and systems [30], but must occur for a limited number of times, preferably once or twice ${ }^{2} \mathrm{Or}$ the full array if the angle or direction cannot be determined or is irrelevant

OOr any other color representing a neutral state

${ }^{4}$ Illumination represent values, that is brighter or dimmer for higher or lower values, respectively

For instance, if three tangibles are nearby/stacked, represented by color $x, y$, and $z$, then all tangibles will use alternating colors $x-y-z$

Table 1. Edge feedback on direct/indirect tangible interactions. to see when on the table. Low-resolution edge display augmented tangibles address this by providing feedback directly on the tangibles that is visible even when tangibles are stacked.

\section{Feedback Mapping}

Table 1 proposes a guideline for mapping different types of edge feedback to different tangible interactions. This guideline is inspired by Harrison et al. [10] and Xu and Lyons's [30] findings and current practices in consumer products, e.g., $[1,9]$, and has been evaluated through a series of informal lab tests. Apart from notifications on direct interactions though tap, touch, tilt, shake, flip, 


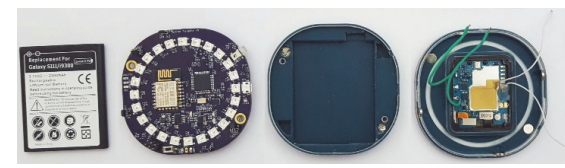

Figure 3. Components of the custom active tangibles from left to right: battery custom printed circuit board with 24-LED ring, bottom of case, top of case including smartwatch.

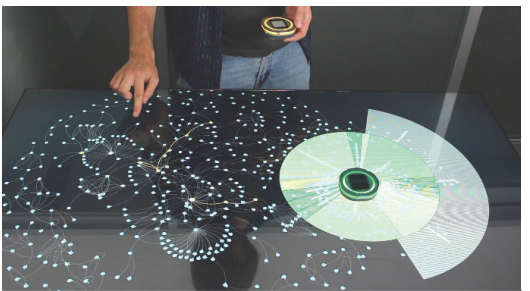

Figure 4. A user exploring gene networks with low-resolution edge display augmented active tangibles using the Sparse Tangibles [3] system. rotate, and tactile buttons on the tangibles, and indirect interactions thorough another device or system, Table 1 also provides guidelines on how to notify the users of a valid or an invalid group interaction, such as stacking and neighboring. Figure 2 illustrates several types of edge feedback from the table.

\section{Demonstration}

We demonstrate how augmenting a low-resolution edge display to an existing system increases its functionality, usability, and provide users with a better understanding of the system.

Custom active tangibles. We designed and developed custom active tangibles, called Actibles [7], using different components of an LG G Watch, a custom circuit board, and a 3D printed case. Actibles include a $41.91 \mathrm{~mm}$ IPS LCD displays $(37.9 \times 46.5 \times 9.95 \mathrm{~mm}), 4$ GB internal storage, 512 MB RAM, and a 9-axis sensor. They run on the Android Wear OS. The custom circuit board contains 8 omnidirectional hall effect sensors for neighboring and stacking detection, an ESP8266 Wi-Fi chip, and an array of 24 LEDs in a circular pattern (Figure 3). They control all inertial sensors using an 8bit microcontroller. Any device, such as a tabletop, can communicate with Actibles using a simple TCP/IP server.

Interactive tabletop. A MultiTaction [18], $1397 \mathrm{~mm}$, $1209.6 \times 680.4 \mathrm{~mm}$ touchscreen area at $1920 \times 1080-$ pixel resolution, placed horizontally on a custom stand was used as an interactive tabletop. It detected the tangibles using its default 2D fiducial marker tracker.

Sparse Tangibles. We picked Sparse Tangibles [3], an existing tabletop and tangible system to demonstrate the proposed feedback mapping. Sparse Tangibles enables collaborative exploration of gene and protein networks on an interactive tabletop using active tangibles (Figure 4). Originally, it used smartwatches with a custom case as active tangibles. Its custom tangibles were also augmented with LED arrays, but did not use it to provide feedback. We picked this system for our demonstration because it utilizes a wide range of on/off-the-table tangible interactions, including tap, multi-touch, shake, and stacking, which makes it ideal for demonstrating different types of edge feedback.

Tangible interactions. The system enables users to select an organism or a gene network and construct expressive queries using active tangibles. To load a network on the tabletop, the user first navigates to the intended organism/gene by performing vertical swipes on the tangible touchscreen, selects the item by tapping, and then places the tangible on the tabletop. To perform a query on the loaded network, the user picks up another tangible, selects a query parameter, such as hub density, and stacks it on the other tangible/s. This updates the network to display only hubs that meet the selected criteria. The user can remove an item from a tangible by shaking it.

Feedback mapping. We assigned different feedback types to all supported tangible interactions and state changes based on Table 1. We used the colors green and blue to represent organisms and genes, respectively, since they are commonly used in biological network visualization tools. Similarly, we used red and white to represent errors and neutral states.

\section{An Informal Evaluation}

We invited five experienced computational biologists to our lab to interact with the customized Sparse Tangibles 


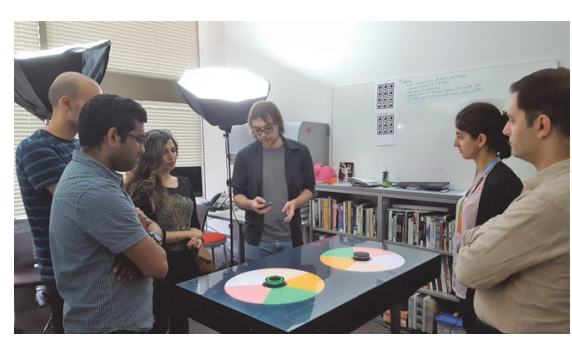

Figure 5. A researcher demonstrating the active tangibles augmented with LED arrays to the participants before the informal evaluation.

\section{Question}

Disagree Neutral Agree

Q1) User Experience $\quad 0 \% \quad 60 \% \quad 40 \%$

Q2) Understandability $\quad 0 \% \quad 40 \% \quad 60 \%$

Q3) Off-the-table $\quad 20 \% \quad 60 \% \quad 20 \%$

Q4) Extensibility $\quad 0 \% \quad 20 \% \quad 80 \%$

Table 2. Results of the informal evaluation. system (Figure 5). They were all employees of a biomedical research center. Their age ranged from 20 to 39 years, on average $29.6(S D=6.8$ ). Three of them were male, and two were female. They all had experience working with biological networks. Three of them responded that they frequently work with biological networks, while two responded that they occasionally do

All participants arrived together. Upon arrival, we demonstrated the system and allowed them to interact with it. Then, they were asked to fill out a short questionnaire involving the following questions: (Q1) edge feedback enhances tabletop and tangible interaction experience, (Q2) provides a better understanding of the system and system events, (Q3) facilitates off-the-table interactions, and (Q4) will be useful in other interactive systems. Table 2 illustrates the results.

Results were mostly neutral or positive. Most participants responded that edge feedback provided a better understanding of the system and system events. Interestingly, most participants responded that edge display did not enhance or reduce their on/off-the-table tangible interaction experience. This is most likely because they were unfamiliar with the system prior to the study. Although we demonstrated the system and allowed them to interact with it, such brief exposure to the system was most probably not enough to fully grasp the functionality of the system, making it difficult for them to compare the experience with and without the edge display. Interestingly, in response to Q4, most participants commented that edge display could be useful in other interactive systems. They most probably responded to this question envisioning edge display in a system they are more familiar with. One participant (female, 31 years), for example, commented that edge display could be useful in a tabletop system that allows tracking filters in clustering alignments. Also, noteworthy that in the demonstration and interactive session, participants used active tangibles augmented with a display screen. They, therefore, responded to the questions in comparison to tangibles that can display visual feedback on the integrated display screens. Nevertheless, participants perceived edge display to be helpful in making the system and events more understandable (Q2). Their responses may have been more optimistic if they used tangibles that do not include a display screen as a baseline.

\section{Conclusion}

We proposed augmenting tangible objects with a lowresolution edge display to provide real-time visual feedback on the current state of the system. We presented a guideline for mapping different types of edge feedback to different tangible interactions. We demonstrate its effectiveness in an informal evaluation that revealed that visual feedback on edge display provides a better understanding of the system.

\section{Future Work}

The informal evaluation did not investigate all potential benefits of the edge display. In the future, we will fully evaluate it in a formal study. We will also extend our guideline to support more actions and explore edge display in the context of smaller tangibles that cannot incorporate 24 LEDs.

\section{Acknowledgements}

This work has been supported in part by NSF-IIS grant 1320350, the Canada Research Chairs program,

NSERC, the Canada Foundation for Innovation, and the Ontario Ministry of Research and Innovation. 


\section{References}

1. Amazon. Amazon Echo. Retrieved January 21, 2017 from https://www.amazon.com/dp/B00X4WHP5E

2. Ahmed Sabbir Arif, Benedikt Iltisberger, and Wolfgang Stuerzlinger. 2011. Extending mobile user ambient awareness for nomadic text entry. In Proceedings of the 23rd Australian Computer-Human Interaction OZCHI'11, 21-30.

https://doi.org/10.1145/2071536.2071539

3. Ahmed Sabbir Arif, Roozbeh Manshaei, Sean Delong, Brien East, Matthew Kyan, and Ali Mazalek. 2016.

Sparse Tangibles: collaborative exploration of gene networks using active tangibles and interactive tabletops. In Proceedings of the TEI '16: Tenth International Conference on Tangible, Embedded, and Embodied Interaction - TEI '16, 287-295.

https://doi.org/10.1145/2839462.2839500

4. Saskia Bakker, Debby Vorstenbosch, Elise van den Hoven, Gerard Hollemans, and Tom Bergman. 2007. Weathergods: tangible interaction in a digital tabletop game. In Proceedings of the 1st international conference on Tangible and embedded interaction TEI '07, 151.

https://doi.org/10.1145/1226969.1227000

5. Christopher Campbell and Peter Tarasewich. 2004. What can you say with only three pixels? Proceedings of the 6th International Symposium on Mobile HumanComputer Interaction - MobileHCI '04: 1-12. https://doi.org/10.1007/978-3-540-28637-0_1

6. Lee Chia-Hsun, Ma Yu-Pin, and Jeng Taysheng. 2003. A spatially-aware tangible interface for computer-aided design. In $\mathrm{CHI}$ '03 extended abstracts on Human factors in computing systems - $\mathrm{CHI}$ '03, 960. https://doi.org/10.1145/765891.766094

7. Brien East, Sean DeLong, Roozbeh Manshaei, Ahmed Sabbir Arif, and Ali Mazalek. 2016. Actibles: open source active tangibles. In Proceedings of the 2016 ACM on Interactive Surfaces and Spaces - ISS '16, 469-472. https://doi.org/10.1145/2992154.2996874

8. Mathieu Le Goc, Pierre Dragicevic, Samuel Huron, Jeremy Boy, and Jean-Daniel Fekete. 2015.
SmartTokens: embedding motion and grip sensing in small tangible objects. In Proceedings of the 28th Annual ACM Symposium on User Interface Software \& Technology - UIST '15, 357-362.

https://doi.org/10.1145/2807442.2807488

9. Google. Google Home. Retrieved January 21, 2017 from https://madeby.google.com/home

10. Chris Harrison, John Horstman, Gary Hsieh, and Scott Hudson. 2012. Unlocking the expressivity of point lights. In Proceedings of the 2012 ACM annual conference on Human Factors in Computing Systems CHI'12, 1683.

https://doi.org/10.1145/2207676.2208296

11. Doris Hausen, Sebastian Boring, Clara Lueling, Simone Rodestock, and Andreas Butz. 2012. StaTube:

facilitating state management in instant messaging systems. In Proceedings of the Sixth International Conference on Tangible, Embedded and Embodied Interaction - TEI '12, 283.

https://doi.org/10.1145/2148131.2148191

12. Marije Kanis, Niall Winters, Stefan Agamanolis, Anna Gavin, and Cian Cullinan. 2005. Toward wearable social networking with iBand. Extended Abstracts Proceedings of the 2005 Conference on Human Factors in Computing Systems: 1521-1524. https://doi.org/10.1145/1056808.1056956

13. Stefanie Klum, Petra Isenberg, Ricardo Langner, JeanDaniel Fekete, and Raimund Dachselt. 2012. Stackables: combining tangibles for faceted browsing. In Proceedings of the International Working Conference on Advanced Visual Interfaces - AVI '12 241. https://doi.org/10.1145/2254556.2254600

14. Gordon Kurtenbach and William Buxton. 1994. User learning and performance with marking menus. In Proceedings of the SIGCHI conference on Human factors in computing systems celebrating interdependence - CHI '94, 258-264. https://doi.org/10.1145/191666.191759

15. Ali Mazalek and Ahmed Sabbir Arif. 2014. Mobilebased tangible interaction techniques for shared displays. In Proceedings of the 16th international conference on Human-computer interaction with 
mobile devices \& services - MobileHCI '14, 561-562. https://doi.org/10.1145/2628363.2645668

16. David Merrill, Jeevan Kalanithi, and Pattie Maes. 2007. Siftables: towards sensor network user interfaces. Proceedings of the 1st international conference on Tangible and embedded interaction - TEI '07: 75. https://doi.org/10.1145/1226969.1226984

17. Jane Meyers, Jeffrey LaMarche, and Michael Eisenberg. 2010. Craftopolis: blending tangible, informal construction into virtual multiuser communities. In Proceedings of the 9th International Conference on Interaction Design and Children - IDC '10, 242. https://doi.org/10.1145/1810543.1810581

18. MultiTaction. MultiTaction. Retrieved January 21, 2017 from https://www.multitaction.com/

19. Joe Natoli. 2014. Feedback: 5 Principles of Interaction Design To Supercharge Your UI (5 of 5) | Give Good UX | Joe Natoli. Retrieved January 17, 2017 from http://www.givegoodux.com/feedback-5-principlesinteraction-design-supercharge-ui-5-5

20. Donghan Park and Hyunseung Choo. 2016. Vibration based tangible tokens for intuitive pairing among smart devices. In Human Aspects of Information Security, Privacy, and Trust, Theo Tryfonas (ed.). Springer International Publishing, 48-56. https://doi.org/10.1007/978-3-319-39381-0_5

21. James Patten and Hiroshi Ishii. 2007. Mechanical constraints as computational constraints in tabletop tangible interfaces. In Proceedings of the SIGCHI conference on Human factors in computing systems CHI '07, 809.

https://doi.org/10.1145/1240624.1240746

22. Esben Warming Pedersen and Kasper Hornbæk. 2011. Tangible bots: interaction with active tangibles in tabletop interfaces. In Proceedings of the 2011 annual conference on Human factors in computing systems CHI'11, 2975. https://doi.org/10.1145/1978942.1979384

23. Jun Rekimoto, Brygg Ullmer, and Haruo Oba. 2001. DataTiles: a modular platform for mixed physical and graphical interactions. In Proceedings of the SIGCHI conference on Human factors in computing systems CHI '01, 269-276.

https://doi.org/10.1145/365024.365115

24. Jan Richter, Bruce $\mathrm{H}$. Thomas, Maki Sugimoto, and Masahiko Inami. 2007. Remote active tangible interactions. In Proceedings of the 1st international conference on Tangible and embedded interaction TEI '07, 39.

https://doi.org/10.1145/1226969.1226977

25. Peter Tarasewich, Christopher S.Campbell, Tian Xia and Myra Dideles. 2003. Evaluation of visual notification cues for ubiquitous compuiting. Proceedings of 5th International Conference on Ubiquitous Computing(Ubicomp2003): 349-366.

26. Brygg Ullmer, Hiroshi Ishii, and Robert J. K. Jacob. 2005. Token+constraint systems for tangible interaction with digital information. ACM Transactions on Computer-Human Interaction 12, 1: 81-118. https://doi.org/10.1145/1057237.1057242

27. Danli Wang, Yunfeng Qi, Yang Zhang, and Tingting Wang. 2013. TanPro-kit: a tangible programming too for children. In Proceedings of the 12th International Conference on Interaction Design and Children - IDC 13, 344-347. https://doi.org/10.1145/2485760.2485841

28. Amanda Williams, Shelly Farnham, and Scott Counts. 2006. Exploring wearable ambient displays for social awareness. Extended Abstracts on Human Factors in Computing Systems (CHI'06): 1529-1534 https://doi.org/10.1145/1125451.1125731

29. Andy Wu, Jung-bin Yim, Eric Caspary, Ali Mazalek, Sanjay Chandrasekharan, and Nancy J Nersessian. 2011. Kinesthetic Pathways: a tabletop visualization to support discovery in systems biology. In Proceedings of the 8th ACM conference on Creativity and cognition - C\&C'11, 21. https://doi.org/10.1145/2069618.2069624

30. Cheng $\mathrm{Xu}$ and Kent Lyons. 2015. Shimmering smartwatches: exploring the smartwatch design space. Proceedings of the 9th International Conference on Tangible, Embedded, and Embodied Interaction TEI '15: 69-76. https://doi.org/10.1145/2677199.2680599 